\title{
A REMARK ON THE APPROXIMATE FIXED-POINT PROPERTY
}

\author{
TADEUSZ KUCZUMOW
}

Received 30 November 2001

We give an example of an unbounded, convex, and closed set $C$ in the Hilbert space $l^{2}$ with the following two properties: (i) $C$ has the approximate fixed-point property for nonexpansive mappings, (ii) $C$ is not contained in a block for every orthogonal basis in $l^{2}$.

\section{Introduction}

In [6], Goebel and the author observed that some unbounded sets in Hilbert spaces have the approximate fixed-point property for nonexpansive mappings. Namely, they proved that every closed convex set $C$, which is contained in a block, has the approximate fixed-point property for nonexpansive mappings (AFPP). This result was extended by Ray [14] to all linearly bounded subsets of $l_{p}, 1<p<\infty$. Next, he proved that a closed convex subset $C$ of a real Hilbert space has the fixed-point property for nonexpansive mappings if and only if it is bounded [15]. The first result of Ray [14] was generalized by Reich [16] (for other results of this type see $[1,2,4,5,7,8,9,10,11,12,13,17,19])$. Reich [16] proved the following remarkable theorem: a closed, convex subset of a reflexive Banach space has the AFPP if and only if it is linearly bounded. Next, Shafrir [18] introduced the notion of a directionally bounded set. Using this concept, he proved two important theorems [18].

(1) A convex subset $C$ of a Banach space $X$ has the AFPP if and only if $C$ is directionally bounded.

(2) For a Banach space $X$, the following two conditions are equivalent: (i) $X$ is reflexive; (ii) every closed, convex, and linearly bounded subset $C$ of $X$ is directionally bounded.

Therefore, the following statements are equivalent: (a) $X$ is reflexive; (b) a closed, convex subset $C$ of $X$ has the AFPP if and only if $C$ is linearly bounded. This result is strictly connected with the above-mentioned Reich theorem [16].

Copyright (C) 2003 Hindawi Publishing Corporation Abstract and Applied Analysis 2003:2 (2003) 93-99 2000 Mathematics Subject Classification: 47H09, 47H10

URL: http://dx.doi.org/10.1155/S108533750320703X 
94 A remark on the approximate fixed-point property

Now, it is worth to note that, recently, there is a return to study the AFPP First, Espínola and Kirk [3] published a paper about the AFPP in the product spaces. They proved that the product space $D=(M \times C)_{\infty}$ has the AFPP for nonexpansive mappings whenever $M$ is a metric space which has the AFPP for such mappings and $C$ is a bounded, convex subset of a Banach space. Next, Wiśnicki wrote a paper about a common approximate fixed-point sequence for two commuting nonexpansive mappings (see [20] for details). Therefore, the author decided to publish an example of a set which is closely related to the AFPP Namely, it is obvious that every blockable set in $l^{2}$ is linearly bounded, but there are linearly bounded sets in $l^{2}$ which are not contained in any block with respect to an arbitrary basis. This was mentioned in [6] but never published. The aim of this paper is to show the construction of such a set.

\section{Preliminaries}

Throughout this paper, $l^{2}$ is real, $\langle\cdot, \cdot\rangle$ denotes the scalar product in $l^{2}$, and $\left\{e_{n}\right\}$ is the standard basis in $l^{2}$.

For any nonempty set $K \subset l^{2}$, the closed convex hull of $K$ is denoted by conv $K$.

Let $C$ be a nonempty subset of a Banach space $X$. A mapping $T: C \rightarrow C$ is said to be nonexpansive if for each $x, y \in C$,

$$
\|T(x)-T(y)\| \leq\|x-y\| .
$$

A convex subset $C$ of a Banach space $X$ has the approximate fixed-point property (AFPP) if each nonexpansive $T: C \rightarrow C$ satisfies

$$
\inf \{\|x-T(x)\|: x \in C\}=0
$$

It is obvious that bounded convex sets always have the AFPP.

A set $K \subset l^{2}$ is said to be a block in the orthogonal basis $\left\{\tilde{e}_{n}\right\}$ if $K$ is of the form

$$
K=\left\{x \in l^{2}:\left|\left\langle x, \tilde{e}_{n}\right\rangle\right| \leq M_{n}, n=1,2, \ldots\right\}
$$

where $\left\{M_{n}\right\}$ is a sequence of positive reals.

The set $C \subset l^{2}$ is called a block set if there exists a block $K \subset l^{2}$ such that $C$ is a subset of $K$.

A subset $C$ of a Banach space $X$ is linearly bounded if $C$ has bounded intersections with all lines in $X$. 


\section{The construction}

Let $\left\{k_{n}\right\}_{n=2}^{\infty}$ and $\left\{l_{n}\right\}_{n=2}^{\infty}$ be two sequences of positive reals such that

$$
\sum_{n=2}^{\infty} \frac{k_{n}}{l_{n}}<+\infty, \quad \lim _{n} k_{n}=+\infty
$$

For example, we may take $k_{n}=n$ and $l_{n}=n^{3}$ for $n=2,3, \ldots$ Next, we set

$$
a_{n}=k_{n} e_{1}+l_{n} e_{n}, \quad b_{n}=-k_{n} e_{1}+l_{n} e_{n},
$$

for $n=2,3, \ldots$, and finally,

$$
C=\operatorname{conv}\left\{x \in l^{2}: \exists n \geq 2\left(x=a_{n} \vee x=b_{n}\right)\right\} .
$$

THEOREM 3.1. If

$$
x=\sum_{n=1}^{\infty} c_{n} e_{n}=c_{1} e_{1}+\sum_{n=2}^{\infty} d_{n} l_{n} e_{n}=c_{1} e_{1}+\bar{x}
$$

is an element of the set $C$, then

$$
d_{n} \geq 0
$$

for $n=2,3, \ldots$,

$$
\sum_{n=2}^{\infty} d_{n} \leq 1
$$

and there exist sequences $\left\{\alpha_{n}\right\}_{n=2}^{\infty}$ and $\left\{\beta_{n}\right\}_{n=2}^{\infty}$ such that

$$
c_{1}=\sum_{n=2}^{\infty}\left(\alpha_{n} k_{n}-\beta_{n} k_{n}\right), \quad \alpha_{n}, \beta_{n} \geq 0, \alpha_{n}+\beta_{n}=d_{n},
$$

for $n=2,3, \ldots$ Additionally, there exists a positive constant $M_{\bar{x}}$ such that

$$
0 \leq\left(\alpha_{n}+\beta_{n}\right) k_{n}=d_{n} k_{n} \leq M_{\bar{x}} \frac{k_{n}}{l_{n}}
$$

for $n=2,3, \ldots$

Proof. Set

$$
\bar{x}=\sum_{n=2}^{\infty} c_{n} e_{n}=\sum_{n=2}^{\infty} d_{n} l_{n} e_{n} .
$$


96 A remark on the approximate fixed-point property

Observe that, there exists a sequence $\left\{x_{j}\right\}_{j=1}^{\infty}$ such that

$$
x=\lim _{j} x_{j}
$$

with

$$
\begin{aligned}
x_{j} & =\sum_{n=2}^{\infty}\left(\alpha_{n j} a_{n}+\beta_{n j} b_{n}\right) \\
& =\sum_{n=2}^{\infty}\left(\alpha_{n j} k_{n}-\beta_{n j} k_{n}\right) e_{1}+\sum_{n=2}^{\infty}\left(\alpha_{n j} l_{n}+\beta_{n j} l_{n}\right) e_{n} \\
& =\sum_{n=2}^{\infty}\left(\alpha_{n j} k_{n}-\beta_{n j} k_{n}\right) e_{1}+\bar{x}_{j} \in C,
\end{aligned}
$$

where

$$
\bar{x}_{j}=\sum_{n=2}^{\infty}\left(\alpha_{n j} l_{n}+\beta_{n j} l_{n}\right) e_{n}, \quad \alpha_{n j}, \beta_{n j} \geq 0, \sum_{n=2}^{\infty}\left(\alpha_{n j}+\beta_{n j}\right)=1 .
$$

Without loss of generality, we can assume that $\left\{\alpha_{n j}\right\}_{j=1}^{\infty}$ and $\left\{\beta_{n j}\right\}_{j=1}^{\infty}$ tend to $\alpha_{n}$ and $\beta_{n}$, respectively, for $n=2,3, \ldots$. Hence, we have

$$
c_{1}=\sum_{n=2}^{m}\left(\alpha_{n} k_{n}-\beta_{n} k_{n}\right)+\lim _{j} \sum_{n=m+1}^{\infty}\left(\alpha_{n j} k_{n}-\beta_{n j} k_{n}\right)
$$

for each $m \geq 2$. On the other hand,

$$
\bar{x}=\lim _{j} \bar{x}_{j}=\lim _{j} \sum_{n=2}^{\infty}\left(\alpha_{n j} l_{n}+\beta_{n j} l_{n}\right) e_{n}
$$

and, therefore, there exists a constant $0<M_{\bar{x}}<+\infty$ such that

$$
\alpha_{n j} l_{n}+\beta_{n j} l_{n} \leq M_{\bar{x}}
$$

for all $n \geq 2$ and $j \in \mathbb{N}$. This implies that

$$
\begin{gathered}
0 \leq \alpha_{n j} k_{n}+\beta_{n j} k_{n}=\left(\alpha_{n j} l_{n}+\beta_{n j} l_{n}\right) \frac{k_{n}}{l_{n}} \leq M_{\bar{x}} \frac{k_{n}}{l_{n}}, \\
0 \leq\left(\alpha_{n}+\beta_{n}\right) k_{n}=d_{n} k_{n} \leq M_{\bar{x}} \frac{k_{n}}{l_{n}},
\end{gathered}
$$

for all $j, n$, and finally,

$$
\begin{aligned}
\sup _{j}\left|\sum_{n=m+1}^{\infty}\left(\alpha_{n j} k_{n}-\beta_{n j} k_{n}\right)\right| & \leq \sup _{j} \sum_{n=m+1}^{\infty}\left(\alpha_{n j} k_{n}+\beta_{n j} k_{n}\right) \\
& \leq \sum_{n=m+1}^{\infty} M_{\bar{x}} \frac{k_{n}}{l_{n}}=M_{\bar{x}} \sum_{n=m+1}^{\infty} \frac{k_{n}}{l_{n}} \stackrel{m \rightarrow \infty}{\longrightarrow}, 0 .
\end{aligned}
$$


Combining (3.13) with (3.17), we conclude that

$$
c_{1}=\sum_{n=2}^{\infty}\left(\alpha_{n} k_{n}-\beta_{n} k_{n}\right) .
$$

This completes the proof.

Theorem 3.2. The set $C$ is linearly bounded but is not a block set in any orthogonal basis in $l^{2}$.

Proof. First, we show that $C$ is not a block set in any orthogonal basis,

$$
\left\{\tilde{e}_{i}\right\}_{i=1}^{\infty}=\left\{\sum_{n=1}^{\infty} c_{i n} e_{n}\right\}_{i=1}^{\infty}
$$

in $l^{2}$. Indeed, there exists $i_{0}$ such that $c_{i_{0}} \neq 0$. Since we have

$$
\max \left(\left|\left\langle a_{n}, \tilde{e}_{i_{0}}\right\rangle\right|,\left|\left\langle b_{n}, \tilde{e}_{i_{0}}\right\rangle\right|\right)=k_{n}\left|c_{i_{0} 1}\right|+l_{n}\left|c_{i_{0} n}\right|
$$

for every $n \geq 2$, these two facts imply that

$$
\sup \left\{\left|\left\langle x, \tilde{e}_{i_{0}}\right\rangle\right|: x \in C\right\}=+\infty \text {. }
$$

Therefore, $C$ is not a block set in $\left\{\tilde{e}_{i}\right\}_{i=1}^{\infty}$.

Now, we prove that the set $C$ is linearly bounded. We begin with the following simple observation:

$$
\sup \left\{\left|\left\langle x, e_{n}\right\rangle\right|: x \in C\right\} \leq l_{n}
$$

for $n=2,3, \ldots$ Next, if $x \in C$ is of the form

$$
x=\sum_{n=1}^{\infty} c_{n} e_{n}=c_{1} e_{1}+\sum_{n=2}^{\infty} d_{n} l_{n} e_{n}=c_{1} e_{1}+\bar{x},
$$

then, by Theorem 3.1, we see that

$$
d_{n} \geq 0
$$

for $n=2,3, \ldots$,

$$
\sum_{n=2}^{\infty} d_{n} \leq 1
$$


and there exist sequences $\left\{\alpha_{n}\right\}_{n=2}^{\infty}$ and $\left\{\beta_{n}\right\}_{n=2}^{\infty}$ such that

$$
c_{1}=\sum_{n=2}^{\infty}\left(\alpha_{n} k_{n}-\beta_{n} k_{n}\right), \quad \alpha_{n}, \beta_{n} \geq 0, \alpha_{n}+\beta_{n}=d_{n},
$$

for $n=2,3, \ldots$. Additionally, there exists a positive constant $M_{\bar{x}}$ such that

$$
0 \leq\left(\alpha_{n}+\beta_{n}\right) k_{n}=d_{n} k_{n} \leq M_{\bar{x}} \frac{k_{n}}{l_{n}}
$$

for $n=2,3, \ldots$ Hence, we obtain

$$
\left|c_{1}\right|=\left|\sum_{n=2}^{\infty}\left(\alpha_{n} k_{n}-\beta_{n} k_{n}\right)\right| \leq \sum_{n=2}^{\infty}\left(\alpha_{n}+\beta_{n}\right) k_{n} \leq M_{\bar{x}} \sum_{n=2}^{\infty} \frac{k_{n}}{l_{n}} .
$$

Then, it follows from (3.22) and (3.28) that an intersection of $C$ with any line $\{y+t v: t \in \mathbb{R}\}$, where $y, v \in l^{2}$ and $v \neq 0$, is either empty or bounded which completes the proof.

\section{References}

[1] A. Canetti, G. Marino, and P. Pietramala, Fixed point theorems for multivalued mappings in Banach spaces, Nonlinear Anal. 17 (1991), no. 1, 11-20.

[2] A. Carbone and G. Marino, Fixed points and almost fixed points of nonexpansive maps in Banach spaces, Riv. Mat. Univ. Parma (4) 13 (1987), 385-393.

[3] R. Espínola and W. A. Kirk, Fixed points and approximate fixed points in product spaces, Taiwanese J. Math. 5 (2001), no. 2, 405-416.

[4] K. Goebel and W. A. Kirk, Topics in Metric Fixed Point Theory, Cambridge Studies in Advanced Mathematics, vol. 28, Cambridge University Press, Cambridge, 1990.

[5] Classical theory of nonexpansive mappings, Handbook of Metric Fixed Point Theory (W. A. Kirk and B. Sims, eds.), Kluwer Academic Publishers, Dordrecht, 2001, pp. 49-91.

[6] K. Goebel and T. Kuczumow, A contribution to the theory of nonexpansive mappings, Bull. Calcutta Math. Soc. 70 (1978), no. 6, 355-357.

[7] K. Goebel and S. Reich, Uniform Convexity, Hyperbolic Geometry, and Nonexpansive Mappings, Monographs and Textbooks in Pure and Applied Mathematics, vol. 83, Marcel Dekker, New York, 1984.

[8] W. A. Kirk, Fixed point theory for nonexpansive mappings, Fixed Point Theory (Sherbrooke, Que., 1980), Lecture Notes in Math., vol. 886, Springer, Berlin, 1981, pp. 484-505.

[9] W. A. Kirk and W. O. Ray, Fixed-point theorems for mappings defined on unbounded sets in Banach spaces, Studia Math. 64 (1979), no. 2, 127-138.

[10] G. Marino, Fixed points for multivalued mappings defined on unbounded sets in Banach spaces, J. Math. Anal. Appl. 157 (1991), no. 2, 555-567.

[11] G. Marino and P. Pietramala, Fixed points and almost fixed points for mappings defined on unbounded sets in Banach spaces, Atti Sem. Mat. Fis. Univ. Modena 40 (1992), no. 1, 1-9. 
[12] J. L. Nelson, K. L. Singh, and J. H. M. Whitfield, Normal structures and nonexpansive mappings in Banach spaces, Nonlinear Analysis, World Scientific Publishing, Singapore, 1987, pp. 433-492.

[13] S. Park, Best approximations and fixed points of nonexpansive maps in Hilbert spaces, Numer. Funct. Anal. Optim. 18 (1997), no. 5-6, 649-657.

[14] W. O. Ray, Nonexpansive mappings on unbounded convex domains, Bull. Acad. Polon. Sci. Sér. Sci. Math. Astronom. Phys. 26 (1978), no. 3, 241-245.

[15] - The fixed point property and unbounded sets in Hilbert space, Trans. Amer. Math. Soc. 258 (1980), no. 2, 531-537.

[16] S. Reich, The almost fixed point property for nonexpansive mappings, Proc. Amer. Math. Soc. 88 (1983), no. 1, 44-46.

[17] J. Schu, A fixed point theorem for nonexpansive mappings on star-shaped domains, Z. Anal. Anwendungen 10 (1991), no. 4, 417-431.

[18] I. Shafrir, The approximate fixed point property in Banach and hyperbolic spaces, Israel J. Math. 71 (1990), no. 2, 211-223.

[19] T. E. Williamson, A geometric approach to fixed points of non-self-mappings $T: D \rightarrow X$, Fixed Points and Nonexpansive Mappings (Cincinnati, Ohio, 1982), Contemp. Math., vol. 18, American Mathematical Society, Rhode Island, 1983, pp. 247253.

[20] A. Wiśnicki, On a problem of common approximate fixed points, preprint, 2001.

Tadeusz Kuczumow: Instytut Matematyki, Uniwersytet M. Curie-Skłodowskiej (UMCS), 20-031 Lublin, Poland; Instytut Matematyki PWSZ, 20-120 Chełm, Poland E-mail address: tadek@golem.umcs.lublin.pl 


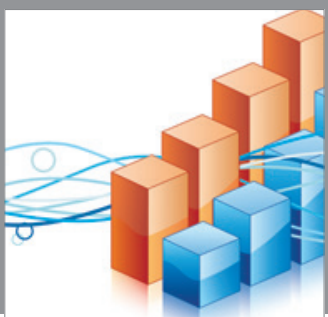

Advances in

Operations Research

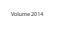

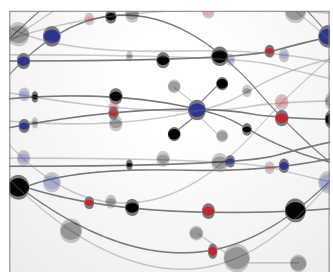

\section{The Scientific} World Journal
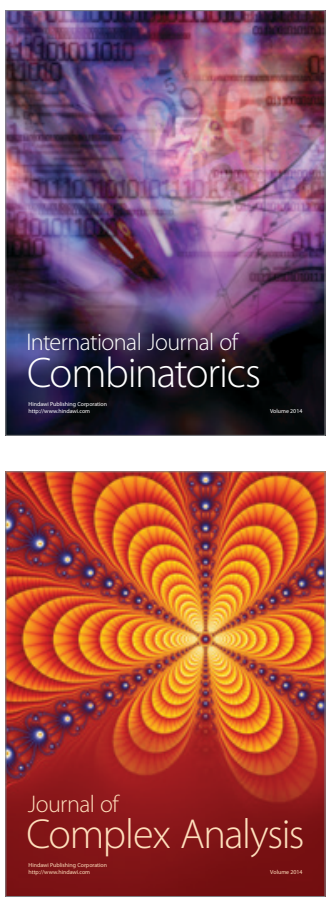

International Journal of

Mathematics and

Mathematical

Sciences
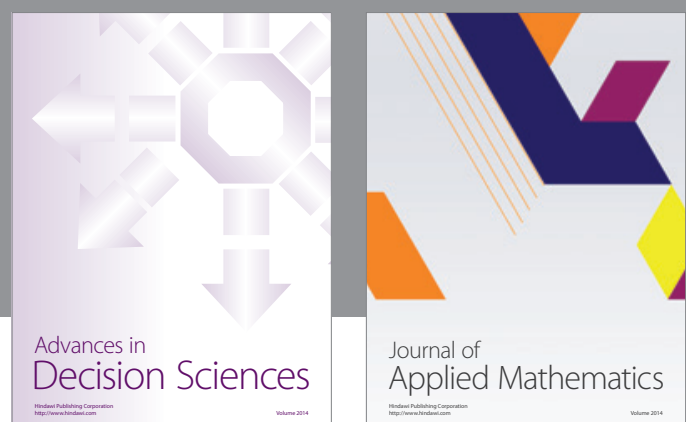

Journal of

Applied Mathematics
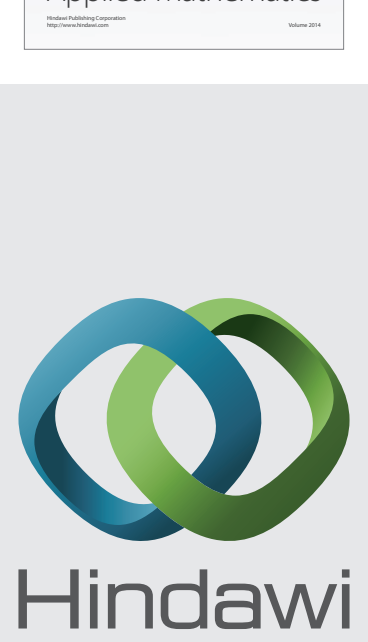

Submit your manuscripts at http://www.hindawi.com
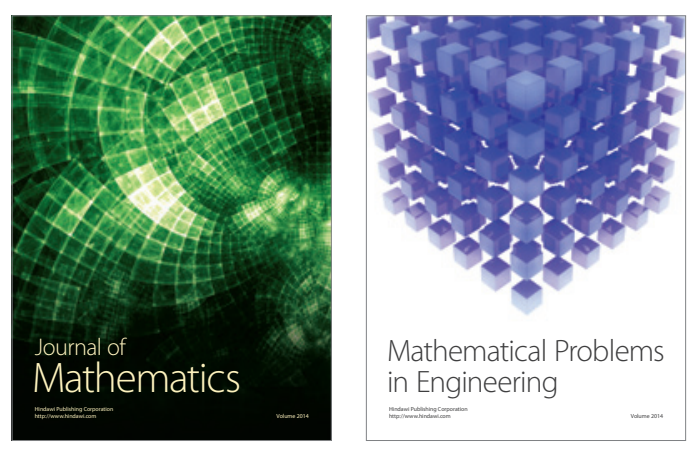

Mathematical Problems in Engineering
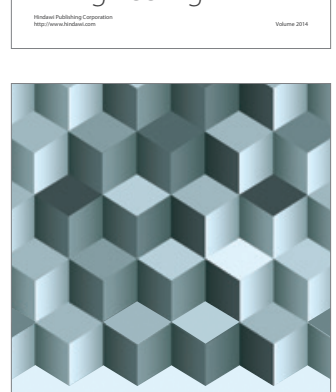

Journal of

Function Spaces
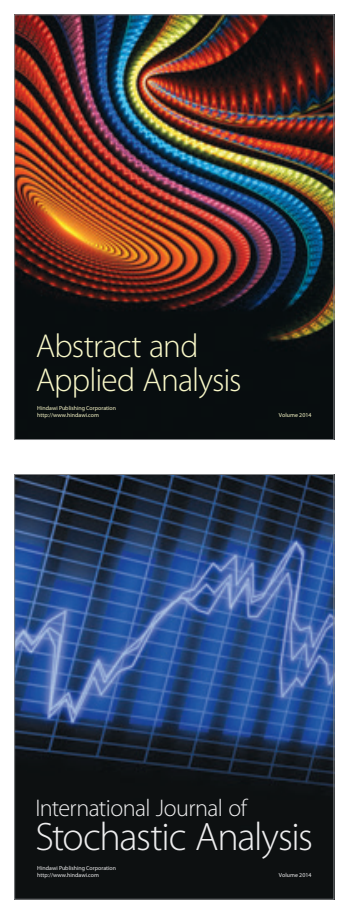

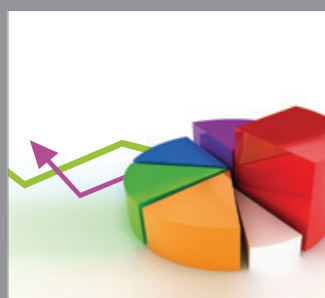

ournal of

Probability and Statistics

Promensencen
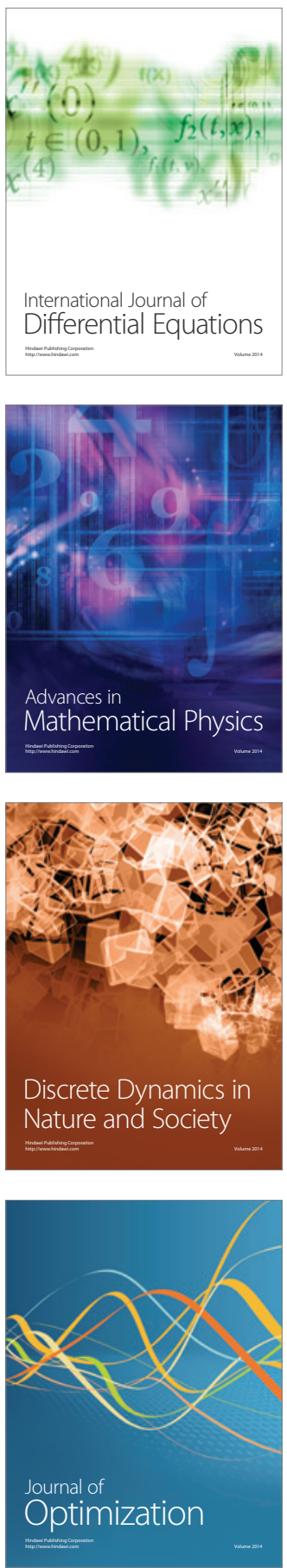